\title{
Correction to: Investigation of lanthanide complexation with acetohydroxamic acid in nitrate medium: experimental and DFT studies
}

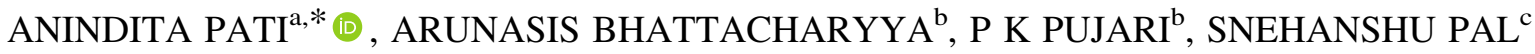 \\ and T K KUNDU ${ }^{\mathrm{a}}$ \\ ${ }^{a}$ Department of Metallurgical and Materials Engineering, Indian Institute of Technology Kharagpur, \\ Kharagpur 721302, West Bengal, India \\ ${ }^{\mathrm{b}}$ Radiochemistry Division, Trombay, Bhabha Atomic Research Centre, BARC, \\ Mumbai 400085, Maharashtra, India \\ ${ }^{\mathrm{c}}$ Department of Metallurgical and Materials Engineering, National Institute of Technology Rourkela, \\ Rourkela 769008, Odisha, India \\ E-mail: patianindita14@gmail.com
}

Correction to: J. Chem. Sci.

https://doi.org/10.1007/s12039-021-01927-0

In this article Snehanshu Pal at affiliation Department of Metallurgical and Materials Engineering, National Institute of Technology Rourkela, Rourkela, Odisha, India-769008 was missing from the author list.
The affiliation Department of Metallurgical and Materials Engineering, Indian Institute of Technology Kharagpur, Kharagpur 721302, West Bengal, India for Author T. K. Kundu was missing.

The original article has been corrected.

*For correspondence 